OSU-HEP-05-06

\title{
Quark and Lepton Masses from Deconstruction
}

\author{
T. Enkhbatt and G. Seidt \\ Department of Physics, Oklahoma State University \\ Stillwater, OK 74078 , USA
}

\begin{abstract}
We propose a supersymmetric $S U(5)^{\prime} \times S U(5)^{\prime \prime}$ model, where the quarks and leptons live in a $U(1)$ product group theory space that is compactified on the real projective plane $R P^{2}$. The fermion generations are placed on different points in the deconstructed manifold by assigning them $S O(10)$ compatible $U(1)$ charges. The observed masses and mixing angles of quarks and leptons emerge from non-renormalizable operators involving the chiral link fields. The link fields introduce a large atmospheric neutrino mixing angle $\theta_{23} \sim 1$ via a dynamical realization of the seesaw mechanism, which sets the deconstruction scale to a value of the order the usual $B-L$ breaking scale $\sim 10^{14} \mathrm{GeV}$. Supersymmetry breaking can be achieved through topological effects due to a nontrivial first homology group $Z_{2}$. The mixed anomalies of the link fields are canceled by Wess-Zumino terms, which are local polynomials in the gauge and link fields only. We also comment on the construction of Chern-Simons couplings from these fields.
\end{abstract}

\footnotetext{
$\dagger$ E-mail: enkhbat@okstate.edu

${ }^{\ddagger}$ E-mail: gerhart. seidl@okstate.edu
} 


\section{Introduction}

There are many reasons why the Standard Model (SM) should be extended. The chief examples to which the SM does not provide any answer are the gauge hierarchy problem, charge quantization, and the origin of fermion masses and mixings. Supersymmetry (SUSY) and four-dimensional (4D) Grand Unified Theories (GUT's) give partial solutions to the first two of the above problems but not to the latter. To understand the observed pattern of fermion masses and mixing angles, it seems therefore necessary that a new ingredient must be added, which allows to distinguish between the generations in a controlled way. For this purpose, one usually advocates a flavor symmetry. Generally, models based on continuous non-Abelian flavor symmetries are highly dependent on the details of the flavor symmetry breaking, without referring to deeper underlying dynamics. The models using an Abelian flavor symmetry, on the other hand, have as a common feature that the three generations carry different charges. At least from a bottom-up point of view, however, generationdependent charges seem to be somewhat contrary to the spirit of GUT's, wherein the ad hoc assignment of hypercharges to the quarks and leptons is explained.

In recent years, higher-dimensional theories opened up new possibilities along this direction $[1,2]$. For example, instead of assuming that the quarks and leptons carry generationdependent charges under a flavor symmetry, the generations might be distinguished by their position in an extra dimension. A hierarchy of Yukawa couplings could then arise from the overlap of the spatial wave-functions of the matter fields in the extra dimension [3]. It would now be interesting to simulate or reproduce this higher-dimensional mechanism in a conventional 4D field theory, which is manifestly gauge-invariant and renormalizable. This can be achieved by employing the idea of dynamically generated extra dimensions, called deconstruction $[4,5]$. In deconstruction 1 one considers the extra dimensions as an infrared effect of an ultraviolet complete theory described by a product of $4 \mathrm{D}$ gauge groups $\Pi_{i} \otimes G_{i}$. The deconstructed dimensions are represented in a "theory space" [7], where the gauge groups $G_{i}$ correspond to "sites" that are connected by "links", like in a transverse lattice gauge theory [8]. Such a view of extra dimensions has rich theoretical and phenomenological implications covering studies in different directions and energy scales. These studies include, for example, electroweak symmetry breaking [9], GUT-type of models [10,11], supersymmetry breaking [7,12-14], and fermion masses and mixings [15-17].2 Yet, a realistic deconstructed

\footnotetext{
${ }^{1}$ For an early approach in terms of infinite arrays of gauge theories, see Ref. [6].

${ }^{2}$ Deconstruction has, for example, also been applied to neutrino oscillations [18], the Casimir effect [19],
} 
model, which gives all the observed fermion masses and mixing angles in the framework of a GUT, has not been proposed so far. This is the aim of the present paper.

The 4D product GUT's which exhibit a higher-dimensional correspondence via deconstruction, have the advantage that dangerous proton decay operators can be easily suppressed by discrete symmetries. The doublet-triplet splitting problem, for example, can be solved in a model proposed by Witten [10], which is based on a 4D SUSY SU(5) product GUT that is obtained from deconstruction. In the present paper, we extend this model by a $U(1)^{N}$ theory space. The different generations of quarks and leptons populate this space and are located at different sites in such a way, that the fermion masses and mixings emerge naturally. A simple linear structure of the product group space, corresponding to a single extra dimension, seems to be too restrictive to account for the entire fermion mass and mixing pattern of the SM. Therefore, we start instead with a deconstructed two-dimensional disk, which can be part of an even larger structure, the so called "spider web theory space" introduced in Ref. [7]. It was shown in Ref. [7], that when the spider web theory space is converted into the real projective plane $R P^{2}$, supersymmetry breaking can be viewed as arising from a topological obstruction due to a nontrivial first homology group $H^{1}\left(R P^{2}\right)=Z_{2}$. In spider web theory space, one can therefore simultaneously account for SUSY breaking and the generation of fermion masses and mixings.

To ensure the consistency of our model, we have to address the anomalies associated with the enlarged gauge symmetry in four dimensions. Anomaly-cancelation in theory space has been previously discussed in Refs. [14, 16, 23, 24]. The cancelation of the anomalies is generally carried out by introducing appropriate Wess-Zumino terms [25], which represent non-decoupling effects of heavy fermions in the low-energy theory. We apply this approach to our spider web theory space. In addition, we examine the continuum limit of Chern-Simons terms, which, however, do not contribute to the anomalies.

The paper is organized as follows. In Sec. 2, we present our model. In Sec. 2.1, we review the solution to the doublet-triplet splitting problem in an $S U(5)^{\prime} \times S U(5)^{\prime \prime}$ product GUT. Next, in Sec. 2.2, we introduce our model for the quark and lepton masses based on a $U(1)$ spider web theory space. We also comment in this section on supersymmetry breaking via the nontrivial topology of $R P^{2}$. The generation of the fermion masses and mixings is described in Sec. 3. The predictions for the up-quarks, down-quarks/charged leptons, and neutrinos are presented in Secs. 3.1, 3.2, and 3.3, The anomaly cancelation in our model is discussed in Sec. 4. Finally, we give our conclusions in Sec. 5 .

instantons [20], gravity [21], and calculable models of the "landscape" of string vacua [22]. 


\section{Deconstructed $U(1)$}

It has been proposed by Witten, that the doublet-triplet splitting problem can be solved in an $4 D$ SUSY $S U(5)^{\prime} \times S U(5)^{\prime \prime}$ product GUT model, which arises from deconstruction [10] (a similar approach has been given earlier by Barbieri et al. [26]). In this section, we will build upon this setup and extend it to a model, which reproduces the observed fermion masses and mixings. We will first begin in Sec. 2.1 with a brief review of the known solution to the doublet-triplet splitting problem, which we then take in Sec. 2.2 as a starting point for introducing our model of quark and lepton masses.

\subsection{Doublet-triplet splitting in $S U(5)^{\prime} \times S U(5)^{\prime \prime}$}

Following the doublet-triplet splitting mechanism proposed by Witten and Barbieri et al., one assumes a $4 \mathrm{D}$ gauge group $G=S U(5)^{\prime} \times S U(5)^{\prime \prime}$, in which the $\mathrm{SM}$ gauge group $G_{S M}=$ $S U(3)_{C} \times S U(2)_{L} \times U(1)_{Y}$ is embedded as a diagonal subgroup. The model possesses a discrete global symmetry $F=Z_{N}$ which commutes with $G$. At the GUT scale, the symmetry group $G \times F$ is spontaneously broken down to $G_{S M} \times F^{\prime}$, where $F^{\prime}$ is a linear combination of $F$ and the $Z_{N}$ subgroup of the hypercharge subgroup $U(1)_{Y}^{\prime \prime}$ of $S U(5)^{\prime \prime}$. The MSSM Higgs doublets are contained in the $S U(5)^{\prime} \times S U(5)^{\prime \prime}$ representations

$$
(\mathbf{5}, \mathbf{1})^{H}+(\mathbf{1}, \overline{\mathbf{5}})^{H}
$$

i.e., the Higgs superfield that gives masses to the up quarks transforms under the fundamental representation of $S U(5)^{\prime}$ and is a singlet under $S U(5)^{\prime \prime}$. The Higgs which generates the down quark and charged lepton masses, on the other hand, is in the antifundamental representation of $S U(5)^{\prime \prime}$ and is an $S U(5)^{\prime}$ singlet. Under $G \supset G_{S M}$, the Higgs fields in Eq. (1) decompose as $\mathbf{5}^{H}=(Q, H)$ and $\overline{\mathbf{5}}^{H}=(\tilde{Q}, \tilde{H})$, in which $H$ and $\tilde{H}$ are the MSSM Higgs doublets, whereas $Q$ and $\tilde{Q}$ are their corresponding color triplet partners. The crucial point which now allows to solve the doublet-triplet splitting problem is here that the unbroken discrete symmetry $F^{\prime}$ commutes with $S U(5)^{\prime}$ but not with $S U(5)^{\prime \prime}$. As a result, $F^{\prime}$ acts on the whole multiplet $5^{H}$ but distinguishes in $\overline{5}^{H}$ between the triplet and doublet components $\tilde{Q}$ and $\tilde{H}$. One can therefore have an $F^{\prime}$-invariant coupling $\tilde{Q} Q$ in the superpotential while a $\mu$-term-type coupling $\sim H \tilde{H}$ is (at the GUT scale) forbidden by $F^{\prime}$, which solves the doublet-triplet splitting problem.

When including quarks and leptons in this model, it is necessary that $F^{\prime}$ can forbid all dangerous baryon number violating operators, which would otherwise mediate proton decay. 
This becomes indeed possible [10], when we assume that under $S U(5)^{\prime} \times S U(5)^{\prime \prime}$ the matter superfields transform as

$$
(\mathbf{1 0}, \mathbf{1})_{i}+(\overline{\mathbf{5}}, \mathbf{1})_{i}+(\mathbf{1}, \mathbf{1})_{i}
$$

where the subscript $i=1,2,3$ is the generation index. In other words, we suppose that the SM quarks and leptons are in non-trivial representations of the first factor $S U(5)^{\prime}$ and singlets under $S U(5)^{\prime \prime}$. Notice in Eq. (2), that we have completed each generation by one "right-handed" (i.e., SM singlet) neutrino required to obtain small neutrino masses via the type-I seesaw mechanism [27]. Since the down quark and charged lepton masses can thus only emerge from non-renormalizable operators, this may provide a reason why the down quarks and charged leptons are generally lighter than the up quarks. Apart from this generic property, however, it would be desirable to have in this model a more complete understanding of the observed masses and mixings of quarks and leptons. For this purpose, we will in the next section attempt to associate the observed fermion masses and mixing angles with the coupling of the Higgs and matter fields in Eqs. (11) and (2) to the theory space of a deconstructed $U(1)$ symmetry.

\section{2 $U(1)$ theory space}

To address the fermion mass hierarchy in the model reviewed in Sec. 2.1, we will assume that the matter fields "live" in a $U(1)$ product group theory space, which describes a deconstructed manifold. The fermion mass hierarchy arises from placing the different generations in Eq. (21) on distinct points in the deconstructed manifold. Although there may be many possibilities, we will first confine ourselves to a theory space, which is topologically a two-dimensional disk. The reason for our choice is that a supersymmetry breaking mechanism can be made readily available in such a theory space [7]. We comment on a possible implementation of this idea in our model at the end of this section. Our deconstructed manifold is conveniently represented by the "moose" [28] or "quiver" [29] diagram in Fig. 1, which describes a spider web theory space, that is topologically equivalent with a two-dimensional disk. The center of the spider web theory space is surrounded by $k$ concentric circles. Each such circle is defined by $N$ sites and each site $i$, where $i=0,1,2, \ldots, k N$, symbolizes one $G_{i} \equiv U(1)_{i}$ gauge group. The total gauge group of our model is therefore $S U(5)^{\prime} \times S U(5)^{\prime \prime} \times U(1)^{k N+1}$ where $U(1)^{k N+1} \equiv \prod_{i=0}^{k N} U(1)_{i}$. For definiteness, we have in Fig. 1 depicted the case $k=2$ and have explicitly labeled only the sites in the inner part of the disk. When compactifying the disk later on the real projective plane $R P^{2}$, we will require that $N=4 m$, where $m$ is 


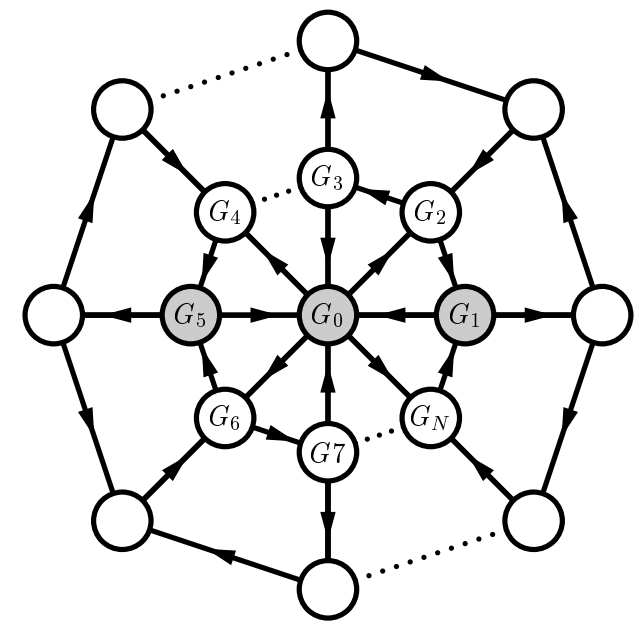

Figure 1: Spider web theory space for the deconstructed $U(1)$ gauge theory. Each point or site $i$, where $i=0,1, \ldots, 2 N$, is associated with a gauge group $G_{i} \equiv U(1)_{i}$. An arrow which connects two groups $G_{i}$ and $G_{j}$ and points from $i$ to $j$ denotes a single chiral link superfield $\phi_{i, j}$ that is charged under $G_{i} \times G_{j}$ as $(+1,-1)$ and is a singlet under all the other gauge groups. The first, second and third generations are placed on the the sites corresponding to $G_{1}, G_{5}$, and $G_{0}$, respectively (gray circles). For $N$ even, the disk is fitted together by triangular plaquettes with alternating orientations. The dotted lines represent possible insertions of extra $U(1)_{i}$ gauge groups.

some integer. In our spider web theory space, two neighboring sites are connected by a single directed link. The general organization of the links and their directions is summarized in Fig. 1 for the example of $k=2$. In Fig. 1, an arrow connecting two sites $i$ and $j$ that points from $i$ to $j$ denotes a chiral link superfield $\phi_{i, j}$, which is charged under $G_{i} \times G_{j}$ as $(+1,-1)$. Under all the other $U(1)$ factors and $S U(5)^{\prime} \times S U(5)^{\prime \prime}$, however, the link fields $\phi_{i, j}$ transform only trivially.

It is important to note in our model that for even $N$, the directions of the link fields in the spider web theory space are such that each small triangular or quadratic plaquette has a definite orientation. Any two neighboring plaquettes have, consequently, opposite orientations. With a single directed link superfield connecting two neighboring sites, only this kind of configuration allows to have Wilson-loop-type contributions (in the sense of usual lattice gauge theory) to the superpotential from every plaquette in Fig. 1. As we will see later, the directions of the link fields are crucial for generating a realistic hierarchy of Yukawa couplings. In what follows, we are interested in the $D$-flat directions $\left|\phi_{0, i}\right|=\left|\phi_{i, i+1}\right| \equiv v$ $(i=1, \ldots, N)$ in the classical moduli space of vacua: All scalar components of the chiral link superfields have vacuum expectation values (VEV's) with a universal magnitude $v$. Such a 
VEV $v$ breaks the $U(1)$ product gauge group spontaneously down to the diagonal subgroup $U(1)_{\text {diag. }}$. Henceforth, we will refer to the field theory defined by our spider web theory space also as the " $U(1)$ theory space" of our model.

Let us now describe how the three generations are incorporated in our theory space. We suppose that each generation in Eq. (2) is put on a separate site (see Fig. 11): the first generation "lives" on site 1 , the second on site 5 , and the third on site 0 in the center of the $\operatorname{disk} 3$ This is achieved by giving the first, second and third generations nonzero $U(1)$ charges exactly under the gauge groups $U(1)_{1}, U(1)_{5}$, and $U(1)_{0}$, respectively, while we assume that they are singlets under all the other $U(1)$ factors. Next, we have to specify on the three sites the $U(1)$ charge assignment to the matter fields within each generation. We choose the $U(1)$ charges for the fermions in each generation to be compatible with $S O(10)$ as follows

$$
\begin{array}{ll}
S O(10) \supset S U(5)^{\prime} \times U(1)_{1}: & \mathbf{1 6}_{1}=\mathbf{1 0}(-1)_{1}+\overline{\mathbf{5}}(3)_{1}+\mathbf{1}(-5)_{1}, \\
S O(10) \supset S U(5)^{\prime} \times U(1)_{5}: & \mathbf{1 6}_{2}=\mathbf{1 0}(1)_{2}+\overline{\mathbf{5}}(-3)_{2}+\mathbf{1}(5)_{2}, \\
S O(10) \supset S U(5)^{\prime} \times U(1)_{0}: & : \mathbf{1 6}_{3}=\mathbf{1 0}(1)_{3}+\overline{\mathbf{5}}(-3)_{3}+\mathbf{1}(5)_{3},
\end{array}
$$

where the parenthesis contains the the corresponding $U(1)_{i}$ charge of the multiplets and the subscript denotes the generation index. In Eqs. (3), we have, as compared to Eq. (22), only kept the transformation properties of the matter fields under $S U(5)^{\prime}$ since they all transform trivially under $S U(5)^{\prime \prime}$. Note also in Eq. (3a), that we have made use of an overall sign ambiguity in the branching rule and assumed that the $U(1)_{1}$ charges of the first generation are "flipped" with respect to the corresponding $U(1)_{5}$ and $U(1)_{0}$ quantum numbers in Eqs. (3b) and (3c). We emphasize that the two lighter generations are connected to the third generation by link fields $\phi_{1,0}$ and $\phi_{5,0}$, which point toward the center. This orientation is crucial for generating realistic fermion masses and mixings.

At this point, it is important to emphasize that we employ in Eqs. (3) the $S O(10)$ branching rules only as a mere guideline or organizing principle for the $U(1)$ charge assignment to the multiplets in Eq. (21). Our model does therefore not possess an actual $S O(10)$ gauge symmetry (for recent flavor discussions in $S O(10)$ models see, e.g., Ref. [30]). Choosing the $S O(10)$ branching rules as a prescription for the $U(1)$ charge assignment, however, has several attractive features. One obvious advantage is, e.g., that the quark and lepton sectors are automatically anomaly-free, such that the discussion of anomalies is restricted to the Higgs and link fields only.

\footnotetext{
${ }^{3}$ Instead of putting the second generation on site 5, we could also choose any site $i$ on the boundary as long as $i$ is odd for the desired link direction of $\phi_{i, 0}$ and $i, N-i \geq 5$.
} 
One major feature of our model is that the fermion mass hierarchy is due to the "location" of the different generations on distinct points in theory space (up to the overall sign ambiguity of the $U(1)$ generators $[c f$. Eqs. (3) $]$ ). This is different, e.g., from usual anomalous $U(1)$ models [31] (for recent related works see, e.g., Refs. [32,33]), where the fermion mass hierarchy is understood in terms of flavor-dependent charges under a single $U(1)$. Notice, that the $U(1)$ charge assignment in Eqs. (3) resembles a gauged $B-L$ symmetry [34], whose deconstruction has been discussed in Ref. [16].

Next, let us consider how the Higgs fields in Eq. (1) couple to the $U(1)$ theory space. The $U(1)$ charge assignment to the third generation in Eq. (3C) already fixes the transformation properties of $(\mathbf{5}, \mathbf{1})^{H}$. Specifically, to obtain a large top Yukawa coupling in our model, we suppose that $(\mathbf{5}, \mathbf{1})^{H}$ carries a $U(1)_{0}$ charge -2 and is a singlet under all the other $U(1)$ gauge groups. The Higgs field $(\mathbf{5}, \mathbf{1})^{H}$ is therefore located as a site variable together with the third generation on the center of the disk. It is interesting to note, that the $U(1)_{0}$ charge assignment to $\mathbf{5}^{H}$ becomes compatible with $S O(10)$ when considering the $\mathbf{5}^{H}$ as part of the decomposition $\mathbf{1 0}^{H}=\mathbf{5}^{H}(-2)+\overline{\mathbf{5}}^{H}(2)$ under $S O(10) \supset S U(5)^{\prime} \times U(1)_{0}$, which is also in agreement with the choice of the $U(1)_{0}$ generator in Eq. (3c). All matter and Higgs superfields which are located on the disk have in common, that they are singlets under the second factor $S U(5)^{\prime \prime}$. In contrast to this, we assume that $(\mathbf{1}, \overline{\mathbf{5}})^{H}$ in Eq. (1), which is the only non-trivial $S U(5)^{\prime \prime}$ representation, carries no $U(1)$ charge at all and is thus not part of the $U(1)$ theory space.

In order to break $U(1)_{\text {diag, }}$, which is not observed at low energies, we assume a single vectorlike pair of chiral superfields $f$ and $\bar{f}$, which resides on the center of the disk. The fields $f$ and $\bar{f}$ are charged under $U(1)_{0}$ as +1 and -1 , respectively, and are singlets under all the other $U(1)$ and $S U(5)$ gauge groups. In what follows, we will suppose that the scalar components of the fields $f$ and $\bar{f}$ acquire VEV's $\langle f\rangle \simeq\langle\bar{f}\rangle \simeq\left\langle\phi_{i, j}\right\rangle \simeq v$, i.e., it is assumed that all $U(1)_{i}$ symmetries including $U(1)_{\text {diag }}$ are broken around the same scale $v$.

As mentioned earlier, we have an interesting possibility of supersymmetry breaking in spider web theory space. Supersymmetry breaking can be implemented in a number of different ways for our case. Among these we find the mechanism discussed in Ref. [7] to be attractive and unique in deconstruction. In the remainder of this section, we will briefly comment on this mechanism.

In Ref. [7], different types of theory space are shown to preserve supersymmetry only locally, viz., the interactions on each plaquette are manifestly supersymmetric. If, however, the topology of theory space has a nontrivial first homology group, supersymmetry breaking 
can be seen as a topological effect. A deconstructed manifold with this property can, e.g., be obtained from the disk in Fig. 1, when we identify diametrically opposite sites and links on the boundary, which yields a real projective plane $R P^{2}$ with first homology group $Z_{2}$ (this requires in our case $N=4 m$, where $m$ is some integer). The phase differences between the gauge couplings $g_{i}$ associated with the gauge groups $U(1)_{i}$ and the corresponding gaugeYukawa couplings $h_{i}=g_{i} e^{i \theta_{i}}$ for the interaction $\sim h_{i} \psi^{\dagger} \lambda_{i} \phi$ (where $\psi$ and $\phi$ denote the fermionic and scalar components of a link field connected to the site $i$ with gaugino $\lambda_{i}$ ), can be removed separately in each plaquette by field redefinitions. In this sense, supersymmetry is preserved "locally". Globally, however, there can remain one phase in the product of all the couplings $h_{i}$, which cannot be rotated away. On $R P^{2}$, this phase is either +1 , which will lead to exact global supersymmetry, or -1 for maximal supersymmetry breaking [7]. The phase being -1 rather than arbitrary, as it would be the case on a circle, can be considered as an advantage of the spider-web theory space. The supersymmetry breaking effects are suppressed by a factor $m_{\text {SUSY }}^{2} \sim \Pi_{i} g_{i}^{2} /(4 \pi)^{2} v^{2}$ (where $i$ runs over half of the boundary of the disk in Fig. 1) due to a number of $N$ loops to account for the nontrivial global twist of $R P^{2}$, which can easily produce a TeV scale supersymmetric spectrum. With this mechanism, one can now address both the fermion mass hierarchy and supersymmetry breaking in the same theory space.

\section{Quark and lepton masses}

\subsection{Up quark sector}

With the representation content outlined in Sec. 2, we are now in a position to determine the fermion masses in our model. Let us first consider the up quark sector. In the notation of Eqs. (11) and (2), the up quark Yukawa couplings arise from $G$-invariant terms of the type $\sim(\mathbf{5}, \mathbf{1})^{H}(\mathbf{1 0}, \mathbf{1})_{i}(\mathbf{1 0}, \mathbf{1})_{j}$ in the superpotential. Depending on the location of the $(\mathbf{1 0}, \mathbf{1})_{i}$ matter multiplets on the disk, these terms may be renormalizable or non-renormalizable. The mass of the top-quark, e.g., emerges from the gauge-invariant renormalizable operator $(\mathbf{5}, \mathbf{1})^{H}(\mathbf{1 0}, \mathbf{1})_{3}(\mathbf{1 0}, \mathbf{1})_{3}$, with a top Yukawa coupling of order one. This coupling is renormalizable because the third generation is situated together with $(\mathbf{5}, \mathbf{1})^{H}$ on the center of the disk carrying $S O(10)$ compatible $U(1)_{0}$ charges.

Since the first two generations are located at some distance away from the center, gaugeinvariance under the deconstructed $U(1)$ requires that all other up quark mass terms come 
from non-renormalizable operators involving the link fields $\phi_{i, j}$, which connect the center with the first two generations. The associated effective Yukawa couplings will thus be suppressed by inverse powers of the cutoff scale $\Lambda$ of the effective theory, thereby producing hierarchical mass and mixing parameters in the fermion sectors. In writing down the Yukawa couplings, it is of great importance that we work in a supersymmetric model, where the particular directions of the link fields as defined in Fig. (1) constrain the allowed renormalizable and non-renormalizable terms due to the holomorphicity of the superpotential. The charm quark mass, e.g., arises dominantly from a non-renormalizable dimension-eight operator of the type $\Lambda^{-4} \phi_{0,4}^{2} \phi_{4,5}^{2}(\mathbf{5}, \mathbf{1})^{H}(\mathbf{1 0}, \mathbf{1})_{2}(\mathbf{1 0}, \mathbf{1})_{2}$, which involves two powers of the link fields $\phi_{0,4}$ and $\phi_{4,5}$. Here, the product of links $\phi_{0,4} \phi_{4,5}$ connects the second generation with the Higgs $(5, \mathbf{1})^{H}$ in the center along the shortest "path" on the disk consistent with the holomorphicity of the superpotential. Similarly, the second and third generations mix via the dimension-six term $\Lambda^{-2} \phi_{0,4} \phi_{4,5}(\mathbf{5}, \mathbf{1})^{H}(\mathbf{1 0}, \mathbf{1})_{3}(\mathbf{1 0}, \mathbf{1})_{2}$ associated with the same path.

Different from the two heavier generations, the mass and mixing terms of the first generation must originate from $U(1)_{\mathrm{diag}}$ violating operators, which involve the $U(1)_{\mathrm{diag}^{-}}$ breaking fields $f$ or $\bar{f}$ that live in the center of the disk. This difference arises because the first generation carries, with respect to the heavier two generations, opposite charges under $U(1)_{\text {diag. }}$. The up quark mass, e.g., is generated by the non-renormalizable term $\Lambda^{-6} f^{4} \phi_{1,0}^{2}(\mathbf{5}, \mathbf{1})^{H}(\mathbf{1 0}, \mathbf{1})_{1}(\mathbf{1 0}, \mathbf{1})_{1}$, involving four powers of $f$. This operator contains also two powers of the link field $\phi_{1,0}$, which connects the first generation with the center. The link field $\phi_{1,0}$ appears therefore also in the operator $\Lambda^{-3} f^{2} \phi_{1,0}(\mathbf{5}, \mathbf{1})^{H}(\mathbf{1 0}, \mathbf{1})_{3}(\mathbf{1 0}, \mathbf{1})_{1}$, which mixes the up with the top quark. Correspondingly, the term $\Lambda^{-5} f^{2} \phi_{1,0} \phi_{0,4} \phi_{4,5}(\mathbf{5}, \mathbf{1})^{H}(\mathbf{1 0}, \mathbf{1})_{1}(\mathbf{1 0}, \mathbf{1})_{2}$ is responsible for the mixing of the up quark with the charm quark. This operator contains the product of links $\phi_{1,0} \phi_{0,4} \phi_{4,5}$, which represents on the disk the shortest connection via holomorphic couplings between the up quark and the charm quark.

In total, the most general gauge-invariant superpotential containing the renormalizable and non-renormalizable terms which are relevant for the up quark masses therefore reads

$$
\begin{aligned}
\mathcal{W} & \supset(\mathbf{5}, \mathbf{1})^{H}(\mathbf{1 0}, \mathbf{1})_{3}(\mathbf{1 0}, \mathbf{1})_{3}+\frac{\phi_{0,4}^{2} \phi_{4,5}^{2}}{\Lambda^{4}}(\mathbf{5}, \mathbf{1})^{H}(\mathbf{1 0}, \mathbf{1})_{2}(\mathbf{1 0}, \mathbf{1})_{2} \\
& +\frac{f^{4} \phi_{1,0}^{2}}{\Lambda^{6}}(\mathbf{5}, \mathbf{1})^{H}(\mathbf{1 0}, \mathbf{1})_{1}(\mathbf{1 0}, \mathbf{1})_{1}+\frac{\phi_{0,4} \phi_{4,5}}{\Lambda^{2}}(\mathbf{5}, \mathbf{1})^{H}(\mathbf{1 0}, \mathbf{1})_{3}(\mathbf{1 0}, \mathbf{1})_{2} \\
& +\frac{f^{2} \phi_{1,0}}{\Lambda^{3}}(\mathbf{5}, \mathbf{1})^{H}(\mathbf{1 0}, \mathbf{1})_{3}(\mathbf{1 0}, \mathbf{1})_{1}+\frac{f^{2} \phi_{1,0} \phi_{0,4} \phi_{4,5}}{\Lambda^{5}}(\mathbf{5}, \mathbf{1})^{H}(\mathbf{1 0}, \mathbf{1})_{1}(\mathbf{1 0}, \mathbf{1})_{2}+\ldots
\end{aligned}
$$

where the dots denote negligible higher-order terms and where we have not explicitly written 
the different Yukawa couplings of order one. When all the link and site fields $\phi_{i, j}$ and $f$ acquire their VEV's around the deconstruction scale $v$, the up quark mass matrix is then given by the well-known texture

$$
M_{u}=\langle H\rangle\left(\begin{array}{ccc}
\epsilon^{6} & \epsilon^{5} & \epsilon^{3} \\
\epsilon^{5} & \epsilon^{4} & \epsilon^{2} \\
\epsilon^{3} & \epsilon^{2} & 1
\end{array}\right),
$$

where we have introduced the small symmetry-breaking parameter $\epsilon \equiv v / \Lambda \simeq 0.2$. Since the texture in Eq. (5) can already fully account for the observed CKM angles, the down quark mixing should not become too large in order to avoid conflict with experiment. As we will see in Sec. 3.2, the mixing in the down sector does indeed not exceed the up quark mixing.

\subsection{Down quark and charged lepton sector}

The construction of the down quark and charged lepton Yukawa coupling terms goes along the same lines as for the up quarks in Sec. 3.1, except for the difference that the matter fields and $(\mathbf{1}, \overline{\mathbf{5}})^{H}$ transform in $G$ under different $S U(5)$ factors. The down quark and charged lepton Yukawa couplings must therefore emerge from $G$-invariant terms $(\overline{\mathbf{5}}, \mathbf{5})^{H}(\mathbf{1}, \overline{\mathbf{5}})^{H}(\mathbf{1 0}, \mathbf{1})_{i}(\overline{\mathbf{5}}, \mathbf{1})_{j}$, where $(\overline{\mathbf{5}}, \mathbf{5})^{H}$ denotes in the doublet-triplet splitting mechanism reviewed in Sec. 2.1 a linear combination of Higgs superfields which transforms under $S U(5)^{\prime} \times S U(5)^{\prime \prime}$ as a bifundamental representation. When $(\overline{\mathbf{5}}, \mathbf{5})^{H}$ acquires its VEV at the GUT scale, $G \times F$ is broken down to the low-energy subgroup $G_{S M} \times F^{\prime}$ (see Refs. [10,26]).

To generate the down quark and charged lepton masses, we assume two such bifundamental Higgs superfields $\Phi_{+}$and $\Phi_{-}$, which are put as site variables on the center of the disk and transform under $S U(5)^{\prime} \times S U(5)^{\prime \prime} \times U(1)_{0}$ as $\Phi_{+} \sim(\overline{\mathbf{5}}, \mathbf{5})^{H}(+2)$ and $\Phi_{-} \sim(\overline{\mathbf{5}}, \mathbf{5})^{H}(-2)$, where in each of the last two expression the second parenthesis contains the value of the $U(1)_{0}$ charge. Under all the other $U(1)_{i}$ groups, $\Phi_{+}$and $\Phi_{-}$transform trivially. By the same arguments as in Sec. 3.1, we then find for the relevant superpotential terms responsible for the down quark charged lepton masses

$$
\begin{aligned}
\mathcal{W} & \supset \frac{\Phi_{+}}{\Lambda^{\prime}}(\mathbf{1}, \overline{\mathbf{5}})^{H}(\mathbf{1 0}, \mathbf{1})_{3}(\overline{\mathbf{5}}, \mathbf{1})_{3}+\frac{\phi_{5,0}^{2} \Phi_{+}}{\Lambda^{2} \Lambda^{\prime}}(\mathbf{1}, \overline{\mathbf{5}})^{H}(\mathbf{1 0}, \mathbf{1})_{2}(\overline{\mathbf{5}}, \mathbf{1})_{2} \\
& +\frac{\phi_{0,2}^{2} \phi_{2,1}^{2} \Phi_{-}}{\Lambda^{4} \Lambda^{\prime}}(\mathbf{1}, \overline{\mathbf{5}})^{H}(\mathbf{1 0}, \mathbf{1})_{1}(\overline{\mathbf{5}}, \mathbf{1})_{1}+\ldots,
\end{aligned}
$$

where the dots denote irrelevant higher-order operators and where we have not explicitly written the Yukawa couplings of order one. In Eq. (6), the scale $\Lambda^{\prime}$ is related to the GUTscale $M_{\mathrm{GUT}} \simeq 10^{16} \mathrm{GeV}$ by $\Lambda^{\prime} \simeq M_{\mathrm{GUT}} / \epsilon^{\prime}$, where $\epsilon^{\prime} \sim 0.1$. Observe that $\Lambda^{\prime}$ is a common 
factor to the down sector parameterizing $\tan \beta$ and thus plays no role for the flavor structure. When $\Phi_{+}$and $\Phi_{-}$acquire similar VEV's $\left\langle\Phi_{+}\right\rangle \simeq\left\langle\Phi_{-}\right\rangle \sim M_{\mathrm{GUT}}$, the mass matrix of the down quarks and charged leptons takes the form

$$
M_{d}=\epsilon^{\prime}\langle\tilde{H}\rangle\left(\begin{array}{ccc}
\epsilon^{4} & \epsilon^{6} & \epsilon^{3} \\
\epsilon^{10} & \epsilon^{2} & \epsilon^{2} \\
\epsilon^{8} & \epsilon^{3} & 1
\end{array}\right),
$$

where the rows and columns are spanned by the $(\mathbf{1 0}, \mathbf{1})_{i}$ and $(\overline{\mathbf{5}}, \mathbf{1})_{j}$, respectively, and where we have a moderate $\tan \beta \equiv\langle H\rangle /\langle\tilde{H}\rangle \sim 10$. In total, one therefore obtains for the quark and charged lepton mass ratios

$$
\begin{aligned}
& m_{u}: m_{c}: m_{t}=\epsilon^{6}: \epsilon^{4}: 1, \\
& m_{d}: m_{s}: m_{b}=\epsilon^{4}: \epsilon^{2}: 1, \\
& m_{e}: m_{\mu}: m_{\tau}=\epsilon^{4}: \epsilon^{2}: 1
\end{aligned}
$$

The CKM angles are of the orders

$$
V_{u s} \sim \epsilon, \quad V_{c b} \sim \epsilon^{2}, \quad V_{u b} \sim \epsilon^{3}
$$

In Eq. (7), we observe that the charged lepton mixing angles are $\lesssim \epsilon^{3}$. The large leptonic mixing angles must therefore be almost entirely generated in the neutrino sector. The neutrino masses and mixing angles will be discussed now.

\subsection{Neutrino masses}

Following the generic approach put forward in Ref. [17], we shall relate the absolute neutrino mass scale to the deconstruction scale via a dynamical realization of the type-I seesaw mechanism, where the inverse lattice spacing $\sim v$ is identified with the usual $B-L$ breaking scale $v \simeq 10^{14} \mathrm{GeV}$. To leading order, the total effective $3 \times 3$ neutrino mass matrix $M_{\nu}$ can thus be written as $M_{\nu}=-M_{D} M_{R}^{-1} M_{D}^{T}$, where, as usually, $M_{D}$ denotes the Dirac neutrino mass matrix and $M_{R}$ is the right-handed Majorana mass matrix. The qualitative difference between $M_{D}$ and $M_{R}$ is, of course, that $M_{D}$ is protected by $G_{S M}$ down to the electroweak scale, while $M_{R}$ can already emerge at the deconstruction scale $v$ through the Yukawa interactions between the right-handed neutrinos (which are vectorial with respect to $G$ ) and the link fields.

When determining $M_{D}$ and $M_{R}$ in the same way like $M_{u}$ and $M_{d}$ in Secs. 3.1 and 3.2 , however, we find that the minimal theory space introduced in Sec. 2.2 would only give 
small neutrino mixing angles. To arrive at a large neutrino mixing, one may deviate from minimality and add extra link fields to our $U(1)$ theory space. Specifically, we assume that each of the directed link superfields $\phi_{i, j}$ defined in Sec. 2.2 is accompanied by a pair of vectorlike chiral link superfields $\chi_{i, j}$ and $\bar{\chi}_{j, i}$ which point into opposite directions and acquire universal VEV's of the order $\left\langle\chi_{i, j}\right\rangle \simeq\left\langle\bar{\chi}_{j, i}\right\rangle \simeq v$. While $\phi_{i, j}$ carries the $G_{i} \times G_{j}$ charges $(+1,-1)$, the fields $\chi_{i, j}$ and $\bar{\chi}_{j, i}$ are charged under $G_{i} \times G_{j}$ as $(+8,-8)$ and $(-8,+8)$, respectively. One can check that the incorporation of the link fields $\chi_{i, j}$ and $\bar{\chi}_{j, i}$ has no effect on our results in Sec. 3.1 and 3.2 for the charged fermion mass ratios and CKM angles summarized in Eqs. (8) . In contrast to this, the extra Yukawa interactions between the $\bar{\chi}_{j, i}$ and the right-handed neutrinos introduce a large off-diagonal term in $M_{R}$, which results in a large atmospheric neutrino mixing angle $\theta_{23} \sim 1$. This is a generalization of the scenario for soft breaking of the $L_{e}-L_{\mu}-L_{\tau}$ lepton number in the right-handed sector [35].

A fully realistic description of bilarge neutrino mixing with normal neutrino mass hierarchy can then be obtained by adding on the sites extra Higgs superfields known from standard realizations of the seesaw mechanism. For example, we can assume an $S U(5)^{\prime} \times S U(5)^{\prime \prime}$ singlet Higgs superfield $(\mathbf{1}, \mathbf{1})^{H}$, which is placed together with the second generation on the site 5. The $(\mathbf{1}, \mathbf{1})^{H}$ carries a charge -10 under $U(1)_{5}$ and is a singlet under all the other $U(1)_{i}$ groups. This $U(1)_{5}$ charge assignment becomes compatible with $S O(10)$ on the site 5 , when we identify $(\mathbf{1}, \mathbf{1})^{H}$ with the $S U(5)^{\prime}$ singlet in the decomposition

$$
S O(10) \supset S U(5)^{\prime} \times U(1)_{5}: \overline{\mathbf{1 2 6}}^{H}=\mathbf{1}^{H}(-10)+\mathbf{1 5}^{H}(6)+\ldots,
$$

where we have only written the subrepresentations relevant for $M_{\nu}$. The $(\mathbf{1}, \mathbf{1})^{H}$ couples to the right-handed neutrinos via a renormalizable term $(\mathbf{1}, \mathbf{1})^{H}(\mathbf{1}, \mathbf{1})_{2}(\mathbf{1}, \mathbf{1})_{2}$, thereby supplementing $M_{R}$ with an additional parameter. Choosing $\left\langle(\mathbf{1}, \mathbf{1})^{H}\right\rangle \simeq \epsilon^{7} v$, the effective neutrino mass matrix comes to the familiar form

$$
M_{\nu}=\epsilon \frac{\langle H\rangle^{2}}{v}\left(\begin{array}{ccc}
\epsilon^{4} & \epsilon^{2} & \epsilon \\
\epsilon^{2} & 1 & 1 \\
\epsilon & 1 & 1
\end{array}\right) .
$$

Taking $\sim 4 \times 10^{-2} \mathrm{eV}$ as the heaviest active neutrino mass, we find from Eq. (10) for the deconstruction scale a value $v \simeq 10^{14} \mathrm{GeV}$, which is of the order the usual $B-L$ breaking scale. In its present form, however, the 2-3 subblock of $M_{\nu}$ in Eq. (10) has a determinant which is much smaller than $\epsilon$, so that the solar mixing angle would be close to maximal.

In order to obtain a large but not maximal solar mixing angle, we can invoke the type-II seesaw mechanism [36], which provides a contribution of order $\sim \epsilon$ to the 2-3 subblock of $M_{\nu}$ 
in Eq. (10), thus suppressing $\theta_{23}$ down to values $\sim \pi / 6$. The type-II seesaw mechanism may be implemented in our model by adding on the center of the disk a pair of conjugate Higgs superfields as site variables, that transform under $S U(5)^{\prime} \times S U(5)^{\prime \prime} \times U(1)_{0}$ as $(\mathbf{1 5}, \mathbf{1})^{H}(6)$ and $(\overline{\mathbf{1 5}}, \mathbf{1})^{H}(-6)$, respectively, but which are singlets under all the other $U(1)_{i}$ gauge groups (for a discussion of phenomenological implications see, e.g., Ref. [37]). The $U(1)_{0}$ charges of these Higgs fields are $S O(10)$ compatible, as can be seen from the branching rule in Eq. (9), by replacing the gauge group $U(1)_{5}$ by $U(1)_{0}$. The superpotential couplings for the type-II seesaw mechanism involve a renormalizable term $M_{15}(\mathbf{1 5}, \mathbf{1})^{H}(\overline{\mathbf{1 5}}, \mathbf{1})^{H}$, where $M_{15}$ is some high mass scale. Now, after integrating out the heavy Higgs fields, the contribution to the 3-3 element of $M_{\nu}$ in Eq. (10) is of the order $\sim \epsilon^{9}\langle H\rangle^{2} / M_{15}$. If $M_{15} \simeq 10^{9} \mathrm{GeV}$, then the total effective neutrino mass matrix $M_{\nu}$ can assume a similar form like in Eq. (10), with the difference that the determinant of the 2-3 subblock is now of the order $\sim \epsilon$. For our choice of parameters, the model can thus lead to a normal active neutrino mass hierarchy

$$
m_{1}: m_{2}: m_{3}=\epsilon: \epsilon: 1
$$

where $m_{1}, m_{2}$, and $m_{3}$ are the active neutrino masses with solar and atmospheric mass squared differences of the orders $\Delta m_{\odot}^{2} \simeq 10^{-4} \mathrm{eV}^{2}$ and $\Delta m_{\text {atm }}^{2} \simeq 10^{-3} \mathrm{eV}^{2}$, respectively. In this case, we then have a small reactor angle $\theta_{13} \sim \epsilon$, a large but not maximal solar angle $\theta_{12} \sim 1$ and a large atmospheric angle $\theta_{23} \sim 1$, which can be maximal. Our model can therefore accommodate current global fits to neutrino oscillation data [38].

\section{Anomaly Cancelation}

Although the $S O(10)$ compatible $U(1)$ charge assignment to the fermions in Eqs. (31) is anomaly-free, the Higgs field sector in its present form would contain anomalies. Note that, in our spider web theory space, any Higgs superfield with anomalous coupling is either a link field $\phi_{i, j}$ or must be situated as a site variable on a single site. The anomalies coming from these site variables may be directly canceled by simply adding in a standard fashion extra fields on the sites where they reside. In contrast to this, we shall now consider the possibility to cancel the pure and mixed anomalies associated with the link fields $\phi_{i, j}$ without introducing any new fields in the low-energy effective theory.

First, we will discuss the pure (i.e., non-mixed) and gauge-gravitational anomalies. When the topology of the spider web theory space in Fig. 1 is that of a disk, the link fields $\phi_{i, j}$ would give rise to pure and gauge-gravitational anomalies on each site of the boundary. 
Interestingly, these anomalies are completely eliminated, when the spider web theory space is, instead, compactified on the real projective plane $R P^{2}$. Observe that the removal of the pure and gauge-gravitational anomalies by compactifying on $R P^{2}$ relies in an essential way on our requirement to have a definite orientation for each small plaquette in Fig. 1. The compactification on $R P^{2}$ alone, however, does not remove the mixed anomalies induced by the link fields.

Now, let us discuss the cancelation of the mixed gauge anomalies. To this end, we add Wess-Zumino (WZ) terms [25], which can be viewed as emerging from integrating out heavy fermions with large Yukawa couplings. The mass scale of these extra fermions is one or two orders of magnitude above the inverse lattice spacing $v \sim 10^{14} \mathrm{GeV}$. In doing so, we follow Refs. [14,23], wherein the case of a deconstructed fifth dimension has been analyzed. Let us consider in Fig. 1 a site $i \neq 0$ which is not in the center (a similar argumentation holds for $i=0)$. The site $i$ is connected to its four neighboring sites $j_{1}, j_{2}, j_{3}$, and $j_{4}$ by the link fields $\phi_{i, j_{1}}, \phi_{i, j_{2}}, \phi_{j_{3}, i}$, and $\phi_{j_{4}, i}$. Note that $\phi_{i, j_{1}}$ and $\phi_{i, j_{2}}$ point from $i$ to $j_{1}$ and $j_{2}$, while $\phi_{j_{3}, i}$ and $\phi_{j_{4}, i}$ point from $j_{3}$ and $j_{4}$ toward the site $i$. The directions of the link fields are a result of the property of our theory space, that two neighboring small plaquettes have alternating orientations. Under an infinitesimal chiral gauge transformation on the site $i$, the vector multiplet $V_{i}$ belonging to the gauge group $U(1)_{i}$ transforms as $V_{i} \rightarrow V_{i}+\mathrm{i}\left(\bar{\Lambda}_{i}-\Lambda_{i}\right)$, where $\Lambda_{i}$ is the gauge parameter. Denoting by $j_{i}^{\mu}$ the chiral current associated with the gauge transformation at the site $i$, we can arrange in the one-loop 3-point function $\left\langle 0\left|T j_{i}^{\mu} j_{k}^{\nu} j_{l}^{\rho}\right| 0\right\rangle$ the anomalies symmetrically among the three involved currents. In a superfield language, the anomalous variation of the link field Lagrangian $\mathcal{L}_{\text {link }}$ corresponding to the gauge transformation $\Lambda_{i}$ can then be written as

$$
\begin{aligned}
\delta_{\Lambda_{i}} \mathcal{L}_{\text {link }}= & -\frac{\mathrm{i}}{12 \pi^{2}} \int d^{2} \theta \Lambda_{i}\left[W_{j_{1}}^{\alpha} W_{\alpha, j_{1}}-2 W_{i}^{\alpha} W_{\alpha, j_{1}}+\left(j_{1} \leftrightarrow j_{2}\right)\right. \\
& \left.-W_{j_{3}}^{\alpha} W_{\alpha, j_{3}}+2 W_{i}^{\alpha} W_{\alpha, j_{3}}+\left(j_{3} \leftrightarrow j_{4}\right)\right]+ \text { h.c. },
\end{aligned}
$$

where $W_{\alpha, i}$ denotes the supersymmetric field strength of the gauge group $U(1)_{i}$. An analogous expression to Eq. (12) holds for the mixed anomalies $\delta_{\Lambda_{0}} \mathcal{L}_{\text {link }}$, associated with a chiral gauge transformation $\Lambda_{0}$ on the site 0 in the center of the theory space. The mixed anomalies $\delta_{\Lambda_{i}} \mathcal{L}_{\text {link }}$ can be canceled in the low-energy effective theory by appropriate WZ terms, which are constructed from local polynomials in the link fields $\phi_{i, j}$ and gauge multiplets $V_{i}$. To 


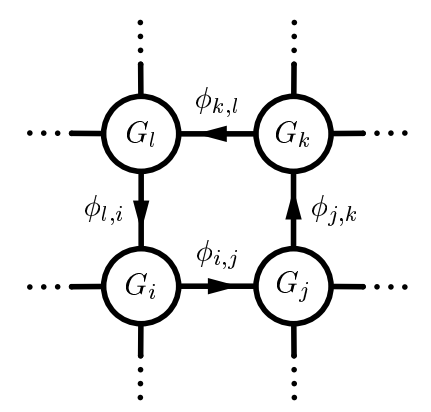

Figure 2: Plaquette in the spider web theory space, considered for the Chern-Simons terms.

remove the mixed anomalies $\delta_{\Lambda_{i}} \mathcal{L}_{\text {link }}$ in Eq. (12), we add to our model the WZ terms

$$
\begin{aligned}
\mathcal{L}_{\mathrm{WZ}}^{i}= & -\frac{1}{24 \pi^{2}} \int d^{2} \theta\left\{\operatorname { l o g } ( \phi _ { i , j _ { 1 } } / v ) \left[\left(C_{1}-1\right) W_{i}^{\alpha} W_{\alpha, i}+\left(C_{1}-1\right) W_{j_{1}}^{\alpha} W_{\alpha, j_{1}}\right.\right. \\
& \left.+\left(C_{1}+2\right) W_{i}^{\alpha} W_{\alpha, j_{1}}\right]+\log \left(\phi_{j_{3}, i} / v\right)\left[\left(C_{1}-1\right) W_{i}^{\alpha} W_{\alpha, i}+\left(C_{1}-1\right) W_{j_{3}}^{\alpha} W_{\alpha, j_{3}}\right. \\
& \left.\left.+\left(C_{1}+2\right) W_{i}^{\alpha} W_{\alpha, j_{3}}\right]\right\}-\frac{C_{2}}{24 \pi^{2}} \int d^{4} \theta\left[\left(V_{i} D_{\alpha} V_{j_{1}}-V_{j_{1}} D_{\alpha} V_{i}\right)\left(W_{i}^{\alpha}+W_{j_{1}}^{\alpha}\right)\right. \\
& \left.\left(V_{i} D_{\alpha} V_{j_{1}}-V_{j_{1}} D_{\alpha} V_{i}\right)\left(W_{i}^{\alpha}+W_{j_{1}}^{\alpha}\right)\right]+\left(j_{1} \leftrightarrow j_{2}\right)+\left(j_{3} \leftrightarrow j_{4}\right)+\text { h.c. },
\end{aligned}
$$

where $C_{1}$ and $C_{2}$ are some suitable parameters. In Eq. (13), the terms with factors $C_{1}$ and $C_{2}$ match in the continuum limit onto six-dimensional (6D) Chern-Simons couplings, when taking the sum of these operators around a plaquette. To see this, let us consider the quadratic plaquette shown in Fig. 2 as a part of the spider web theory space, which is spanned by the sites $i, j, k$, and $l$. From Eq. (13), we find that the sum of all terms with factors $C_{1}$ and $C_{2}$, which correspond to the plaquette, is given by

$$
\begin{aligned}
\mathcal{L}_{\mathrm{CS}}^{i j k l}= & -\frac{C_{1}}{24 \pi^{2}} \int d^{2} \theta \log \left(\phi_{i, j}\right)\left[W_{\alpha, i} W_{i}^{\alpha}+W_{\alpha, j} W_{j}^{\alpha}+W_{\alpha, i} W_{j}^{\alpha}\right] \\
& -\frac{C_{2}}{24 \pi^{2}} \int d^{4} \theta\left[\left(V_{i} D_{\alpha} V_{j}-V_{j} D_{\alpha} V_{i}\right)\left(W_{i}^{\alpha}+W_{j}^{\alpha}\right)\right] \\
& +((i, j) \leftrightarrow(j, k))+((j, k) \leftrightarrow(k, l))+((k, l) \leftrightarrow(l, i))+\text { h.c. },
\end{aligned}
$$

where we have indicated in the last line a cyclic permutation of the four sides of the plaquette. We parameterize the link fields attached to the site $i$ as $\phi_{i, j}=\frac{v}{\sqrt{2}} e^{\left(\Sigma_{i, j}+\mathrm{i} G_{i, j}\right) / v}$ and $\phi_{l, i}=$ $\frac{v}{\sqrt{2}} e^{\left(\Sigma_{l, i}+\mathrm{i} G_{l, i}\right) / v}$. In the continuum limit, $G_{i, j}$ and $G_{l, j}$ become $G_{i, j} \rightarrow A_{5}$ and $G_{l, i} \rightarrow-A_{6}$, where $A_{5}$ and $A_{6}$ are the 5 th and 6 th components of the $U(1)$ gauge field of the $6 \mathrm{D}$ theory. Expanding around the site $i$, the term $\mathcal{L}_{\mathrm{CS}}^{i j k l}$ in Eq. (14) matches in the continuum limit onto

$$
\mathcal{L}_{\mathrm{CS}}^{i j k l} \rightarrow-\frac{1}{24 \pi^{2}} \epsilon^{\mu \nu \rho \sigma}\left[3 C_{1}\left(\partial_{4} A_{5}-\partial_{5} A_{4}\right) \partial_{\mu} A_{\nu} \partial_{\rho} A_{\sigma}-4 C_{2} \partial_{4} A_{\mu} \partial_{5} A_{\nu} \partial_{\rho} A_{\sigma}\right],
$$

which reproduces the $6 \mathrm{D}$ Chern-Simons term $\mathcal{L}_{\mathrm{CS}}=-\left(C_{1} / 8 \pi^{2}\right) \epsilon^{\alpha \beta \mu \nu \rho \sigma}\left[\partial_{\alpha} A_{\beta} \partial_{\mu} A_{\nu} \partial_{\rho} A_{\sigma}\right]$ for the choice $C_{2}=(3 / 2) C_{1}$. To determine the constant $C_{1}$, note in Eq. (13) that the effective 
moduli fields $\log \left(\phi_{i, j}\right)$ transform under gauge transformations on the neighboring sites as $\log \left(\phi_{i, j}\right) \rightarrow \log \left(\phi_{i, j}\right)+2 \mathrm{i}\left(\Lambda_{i}-\Lambda_{j}\right)$. As a consequence, the anomalous variation $\delta_{\Lambda_{i}} \mathcal{L}_{\mathrm{WZ}}^{i}$ of the WZ term in Eq. (13) obeys $\delta_{\Lambda_{i}} \mathcal{L}_{\mathrm{WZ}}=-\delta_{\Lambda_{i}} \mathcal{L}_{\text {link }}$ and thus cancels the mixed anomalies in Eq. (12) when $C_{1}=0$, i.e., the Chern-Simons term has to vanish.

The correspondence to the $6 \mathrm{D}$ model can be established in the infrared limit via the tower of gauge bosons generated by the kinetic term $\sum_{(i, j)}\left(D_{\mu} \phi_{i, j}\right)^{\dagger} D^{\mu} \phi_{i, j}$, in which the covariant derivative $D_{\mu}$ acts on the scalar components of all the links $\phi_{i, j}$ connecting neighboring sites $i$ and $j$. At low energies, the associated gauge boson mass matrix is then schematically given by the contribution $\sim g^{2} v^{2} \sum_{(i, j)}\left(A_{i}^{\mu}-A_{j}^{\mu}\right)^{2}$ to the total Lagrangian, where $A_{i}^{\mu}$ are the $U(1)_{i}$ gauge bosons and where we have, for simplicity, assumed universal gauge couplings for all the $U(1)$ factors and universal VEV's $v$ of the link fields. Although our spider-web theory space is topologically equivalent to the real projective plane $R P^{2}$, a variation of the individual gauge couplings and link field VEV's would offer many possibilities to realize distinct geometries in the continuum limit. At this level, the identification of the exact geometry of the corresponding deconstructed manifold, however, would require further study of the detailed gauge boson spectrum, which is beyond the scope of this paper. Since in our particular fermion mass model the universal inverse lattice spacing is fixed by the neutrino sector to be of the order $v \sim 10^{14} \mathrm{GeV}$, a straightforward way to arrive at a large $N$ limit would be to extend the exterior of the disk in Fig. 1 by simply adding extra quadratic plaquettes to the boundary.

Generally, we wish to point out that the cancelation of the pure and gauge-gravitational anomalies allows also different ways to organize the orientation of the link fields. Consider, for instance, a compactification of the the spider-web theory space on $R P^{n}$ with $n>2$ and even, by partitioning the boundary into $n$ equivalent line segments that are identified under a rotation of the disk by an angle $2 \pi / n$. We can arrive in this case at a similar phenomenology of fermion masses, when in Fig. 1 all links on the concentric circles point, e.g., in clockwise direction while all the radial links within each of the $n$ segments of the disk point either inward or outward but alternate between two adjacent segments.

Finally, one can ask whether our model is embedable into a string construction as suggested by quiver gauge theories [29,39]. Recent advances in the AdS/CFT correspondence [40] show that infinite classes of $4 \mathrm{D} \mathcal{N}=1$ SUSY quiver gauge theories can be viewed as a stack of D3-branes probing a singular Calabi-Yau manifold. For our specific model, however, such embeddings remain to be seen. 


\section{Conclusions}

In this paper, we have presented a model, wherein the observed fermion masses and mixing angles emerge from a deconstructed $U(1)$ theory space. We have extended a supersymmetric $S U(5)^{\prime} \times S U(5)^{\prime \prime}$ product GUT, which has been previously suggested for solving the doublet-triplet splitting problem $[10,26]$, by a deconstructed $U(1)$ theory space with disk structure. The different generations of the SM fermions live at different sites of the disk. Upon breaking the $U(1)$ product group by the link fields around the $B-L$ breaking scale $v \simeq 10^{14} \mathrm{GeV}$, the effective Yukawa couplings and mixing matrices of the fermions are correctly reproduced through non-renormalizable operators. The $U(1)$ charge assignment to the fermions is compatible with $S O(10)$ and, thus, free from anomalies. This is a major difference compared to usual, e.g., anomalous $U(1)$ models, where the SM generations differ by flavor-dependent charges, which appears to be somewhat adhoc from a bottom-up point of view. The neutrino mass matrix receives contributions from both type-I and type-II seesaw mechanisms. Among many possibilities, we have advocated the supersymmetry breaking scenario of Ref. [7], which is unique to deconstructed models. To do so, the original disk theory space is thought to be part of a larger structure, viz., a spider web theory space. When diametrically opposite sites and links on the boundary of this space are identified, we arrive at an $R P^{2}$ manifold with nontrivial first homology group $Z_{2}$. The interactions on each plaquette are here required to be manifestly supersymmetric. The nontrivial global twist of $R P^{2}$ can be viewed as the source of supersymmetry breaking. Thus, both the fermion mass matrix structures and supersymmetry breaking can now be addressed in the same theory space, which we find interesting and economic. The choice of the charges for the link fields, which defines a direction for the links connecting two sites, is such that neighboring plaquettes have alternating orientations. As a consequence, all the sites have the same number of "ingoing" and "outgoing" link fields. This arrangement insures that the contributions to the pure and gravitational anomalies on each site vanish automatically. We cancel the mixed anomalies, along the line of Refs. [14,23], by Wess-Zumino terms, which can be considered as a result of integrating out heavy fermions with masses one or two orders of magnitude above the $B-L$ breaking scale. We have examined possible Chern-Simons terms on a rectangular plaquette, which nevertheless do not play a role in the anomaly cancelations, and have shown that they have a correct $6 \mathrm{D}$ continuum limit.

It would be clearly interesting to develop descriptions of our model based on gauge groups like $S O(10)$ or $E_{6}$ with a universal GUT/deconstruction scale. In possible variations of our 
model one could (as proposed, e.g., in Ref. [16]) also think of shifting anomalies between the gauge groups using a deconstructed version of the anomaly inflow mechanism known from string theory [41].

\section{Acknowledgments}

We would like to thank K.S. Babu, S. Pokorski, and S. Vempati for useful comments and discussions. One of us (G.S.) would like to thank the Fermilab Theory Group for their warm hospitality and support during the stay as a summer visitor, where part of this work was developed. This work was supported by the U.S. Department of Energy under Grant Numbers DE-FG02-04ER46140 and DE-FG02-04ER41306 (T.E. and G.S.).

\section{References}

[1] N. Arkani-Hamed, S. Dimopoulos, and G.R. Dvali, Phys. Lett. B 429 (1998) 263, hep-ph/9803315.

[2] L. Randall and R. Sundrum, Phys. Rev. Lett. 83 (1999) 3370 (1999), hep-ph/9905221.

[3] N. Arkani-Hamed and M. Schmaltz, Phys. Rev. D 61 (2000) 033005, hep-ph/9903417.

[4] N. Arkani-Hamed, A.G. Cohen, and H. Georgi, Phys. Rev. Lett. 86 (2001) 4757, hep-th/0104005.

[5] C.T. Hill, S. Pokorski, and J. Wang, Phys. Rev. D 64 (2001) 105005, hep-th/0104035.

[6] M.B. Halpern and W. Siegel, Phys. Rev. D 11 (1975) 2967.

[7] N. Arkani-Hamed, A.G. Cohen, and H. Georgi, JHEP 0207 (2002) 020, hep-th/0109082.

[8] W.A. Bardeen and R.B. Pearson, Phys. Rev. D 14 (1976) 547; W.A. Bardeen, R.B. Pearson, and E. Rabinovici, Phys. Rev. D 21 (1980) 1037.

[9] N. Arkani-Hamed, A.G. Cohen, and H. Georgi, Phys. Lett. B 513 (2001) 232, hep-ph/0105239; H.C. Cheng, C.T. Hill, S. Pokorski, and J. Wang, Phys. Rev. D 64 (2001) 065007, hep-th/0104179. 
[10] E. Witten, in Proceedings of the 10th International Conference on Supersymmetry and Unification of Fundamental Interactions (SUSY02), DESY, Hamburg, 2002, hep-ph/0201018.

[11] C. Csaki, G.D. Kribs, and J. Terning, Phys. Rev. D 65 (2002) 015004, hep-ph/0107266

D. Cremades, L.E. Ibañez, and F. Marchesano, JHEP 0207 (2002) 009 (2002), hep-th/0201205; T.j. Li and T. Liu, Eur. Phys. J. C 28 (2003) 545, hep-th/0204128; M. Dine, Y. Nir, and Y. Shadmi, Phys. Rev. D 66 (2002) 115001, hep-ph/0206268; S. Rakshit, G. Raz, S. Roy, and Y. Shadmi, Phys. Rev. D 69 (2004) 095006, hep-ph/0309318 C.S. Huang, J. Jiang, and T. Li, Nucl. Phys. B 702 (2004) 109, hep-ph/0407180; C.D. Carone, Phys. Rev. D 71 (2005) 075013, hep-ph/0503069.

[12] M.A. Luty and R. Sundrum, Phys. Rev. D 65 (2002) 066004, hep-th/0105137; T. Kobayashi, N. Maru, and K. Yoshioka, Eur. Phys. J. C 29 (2003) 277, hep-ph/0110117; D.B. Kaplan, E. Katz, and M. Unsal, JHEP 0305 (2003) 037, hep-lat/0206019.

[13] C. Csaki, J. Erlich, C. Grojean, and G.D. Kribs, Phys. Rev. D 65 (2002) 015003, hep-ph/0106044.

[14] E. Dudas, A. Falkowski, and S. Pokorski, Phys. Lett. B 568 (2003) 281, hep-th/0303155.

[15] D.E. Kaplan and T.M.P. Tait, JHEP 0111 (2001) 051, hep-ph/0110126; H. Abe, T. Kobayashi, N. Maru, and K. Yoshioka, Phys. Rev. D 67 (2003) 045019, hep-ph/0205344; G. Seidl, hep-ph/0301044; P.Q. Hung, A. Soddu, and N.K. Tran, Nucl. Phys. B 712 (2005) 325, hep-ph/0410179; C.D. Froggatt, L.V. Laperashvili, H.B. Nielsen, and Y. Takanishi, hep-ph/0309129.

[16] W. Skiba and D. Smith, Phys. Rev. D 65 (2002) 095002, hep-ph/0201056.

[17] K.R.S. Balaji, M. Lindner, and G. Seidl, Phys. Rev. Lett. 91 (2003) 161803, hep-ph/0303245.

[18] T. Hällgren, T. Ohlsson, and G. Seidl, JHEP 0502 (2005) 049, hep-ph/0411312.

[19] F. Bauer, M. Lindner, and G. Seidl, JHEP 0405 (2004) 026, hep-th/0309200. 
[20] C. Csaki, J. Erlich, V.V. Khoze, E. Poppitz, Y. Shadmi, and Y. Shirman, Phys. Rev. D 65 (2002) 085033, hep-th/0110188.

[21] N. Arkani-Hamed, H. Georgi, and M.D. Schwartz, Annals Phys. 305 (2003) 96, hep-th/0210184; N. Arkani-Hamed and M.D. Schwartz, Phys. Rev. D 69 (2004) 104001, hep-th/0302110; M.D. Schwartz, Phys. Rev. D 68 (2003) 024029, hep-th/0303114 T. Gregoire, M.D. Schwartz, and Y. Shadmi, JHEP 0407 (2004) 029, hep-th/0403224.

[22] K. R. Dienes, E. Dudas and T. Gherghetta, hep-th/0412185.

[23] A. Falkowski, H.P. Nilles, M. Olechowski, and S. Pokorski, Phys. Lett. B 566 (2003) 248, hep-th/0212206.

[24] J. Giedt, E. Poppitz, and M. Rozali, JHEP 0303 (2003) 035 (2003), hep-th/0301048; C.T. Hill and C.K. Zachos, Phys. Rev. D 71 (2005) 046002, hep-th/0411157.

[25] J. Wess and B. Zumino, Phys. Lett. B 37 (1971) 95; E. Witten, Nucl. Phys. B 223 (1983) 422.

[26] R. Barbieri, G.R. Dvali, and A. Strumia, Phys. Lett. B 333 (1994) 79, hep-ph/9404278.

[27] P. Minkowski, Phys. Lett. B 67 (1977) 421; T. Yanagida, in Proceedings of the Workshop on the Unified Theory and Baryon Number in the Universe, KEK, Tsukuba, 1979; M. Gell-Mann, P. Ramond, and R. Slansky, in Proceedings of the Workshop on Supergravity, Stony Brook, New York, 1979.

[28] H. Georgi, Nucl. Phys. B 266 (1986) 274.

[29] M.R. Douglas and G. Moore, hep-th/9603167.

[30] K. Matsuda, Y. Koide, T. Fukuyama, and H. Nishiura, Phys. Rev. D 65 (2002) 033008, Erratum-ibid. D 65 (2002) 079904, hep-ph/0108202; T. Fukuyama and N. Okada, JHEP 0211 (2002) 011, hep-ph/0205066; B. Bajc, G. Senjanović, and F. Vissani, Phys. Rev. D 70 (2004) 093002, hep-ph/0402140; S.M. Barr and B. Kyae, Phys. Rev. D 70 (2004) 075005, hep-ph/0407154; H.S. Goh, R.N. Mohapatra, and S. Nasri, Phys. Rev. D 70 (2004) 075022, hep-ph/0408139; K.S. Babu, J.C. Pati, and P. Rastogi, Phys. Rev. D 71 (2005) 015005, hep-ph/0410200; H.D. Kim, S. Raby, and L. Schradin, hep-ph/0411328; C.H. Albright, hep-ph/0502161. 
[31] L.E. Ibanez and G.G. Ross, Phys. Lett. B 332 (1994) 100, hep-ph/9403338; P. Binetruy and P. Ramond, Phys. Lett. B 350 (1995) 49, hep-ph/9412385; P. Binetruy, S. Lavignac, and P. Ramond, Nucl. Phys. B 477 (1996) 353, hep-ph/9601243.

[32] K.S. Babu, T. Enkhbat, and I. Gogoladze, Nucl. Phys. B 678 (2004) 233, hep-ph/0308093; K.S. Babu and T. Enkhbat, Nucl. Phys. B 708 (2005) 511, hep-ph/0406003; K.S. Babu, T. Enkhbat, and B. Mukhopadhyaya, hep-ph/0501079.

[33] H.K. Dreiner, H. Murayama, and M. Thormeier, hep-ph/0312012; P.H. Chankowski, K. Kowalska, S. Lavignac, and S. Pokorski, Phys. Rev. D 71 (2005) 055004, hep-ph/0501071; G.L. Kane, S.F. King, I.N.R. Peddie, and L. Velasco-Sevilla, hep-ph/0504038.

[34] R.E. Marshak and R.N. Mohapatra, Phys. Lett. B 91 (1980) 222.

[35] W. Grimus and L. Lavoura, JHEP 0107 (2001) 045, hep-ph/0105212.

[36] R.N. Mohapatra and G. Senjanović, Phys. Rev. Lett. 44 (1980) 912; Phys. Rev. D 23 (1981) 165; J. Schechter and J.W.F. Valle, Phys. Rev. D 22 (1980) 2227; G. Lazarides, Q. Shafi, and C. Wetterich, Nucl. Phys. B 181 (1981) 287.

[37] A. Rossi, Phys. Rev. D 66 (2002) 075003, hep-ph/0207006.

[38] M. Maltoni, T. Schwetz, M. Tórtola, and J.W.F. Valle, New J. Phys. 6 (2004) 122, hep-ph/0405172.

[39] F. Cachazo, S. Katz and C. Vafa, hep-th/0108120; N.w. Kim, A. Pankiewicz, S.J. Rey and S. Theisen, Eur. Phys. J. C 25 (2002) 327, hep-th/0203080.

[40] J. P. Gauntlett, D. Martelli, J. Sparks and D. Waldram, Adv. Theor. Math. Phys. 8 (2004) 711, hep-th/0403002; S. Benvenuti, S. Franco, A. Hanany, D. Martelli and J. Sparks, JHEP 0506 (2005) 064, hep-th/0411264.

[41] C.G. Callan and J.A. Harvey, Nucl. Phys. B 250 (1985) 427; S.G. Naculich, Nucl. Phys. B 296 (1988) 837; M.B. Green, J.A. Harvey, and G.W. Moore, Class. Quant. Grav. 14 (1997) 47, hep-th/9605033; E. Witten, hep-th/0108165. 